\title{
Index autorum ad Vol. 163
}

\section{Confecit: C. Loeb-Schürch}

(B) $=$ Buchbesp $\Gamma$ echungen - Book reviews - Livres nouveaux $(\mathrm{V})=$ Vortrag - Report Communication

Auerbach, E., 360 Aviner, Z., 273, 278

Balen, A. Th. M. van, v. Van Balen,

A. Th. M. Balik, J., 63 (V) Barraquer, J., 428 (B) Best, M., 235 Beyer, Ch. K., 418 Biesheuvel, K., 2 (V) Bleeker, G. M., 44 (V) Blumenthal, M, 235 Bonomi, L., 347 Brancato, R., 189

Bruckner, R., 185

Comberg, U., 289 Crone, R. A., 15 (V) Cuendet, J. F., 306 Custovic, K., 305

De Haan, A. B., 26 (V) Deller, M., 403 D’Ermo, F., 347 Deufrains, A., 374 Ducrey, N., 254

Dufour, R., 325 Duke-Elder, S., 288 (B)

Feinsod, M., 360 Francois, J., 312 Freund, M., 65 Frühauf, Anneíies, 369

Gailloud, CL, 325 Galin, A., 235 Gliem, H., 216, 411 Gloeckner, S. L., 128 (B) Goder, G., 289

Guntermann, S., 393

Haan, A. B. de, v. De Haan, A. B.

Hagedoorn, A., $27(\mathrm{~V})$

Havener, W. H, 128 (B) Herishanu, Y., 98, 302 Hoogenboom, W. P. H., 209 Hoogerheide, J., 209 Hoppenbrouwers, R., 36 (V), 41 (V)

Johnston, A. W., 102

Kalberer, M, 171 Kietzmann, G., 411 Kolin, J., 46 (V) Krause, U., 136 Kühl, W., 245 Kumar, J., 150

Landry, M., 254

Lansberg, M. P., 41 (V)

Lapis, K., 73

Lavy, S., 302

Leopold, H. I. (ed.), 128 (B)

Lerche, W., 199

Lith, G. H. M. van, v. Van Lith,

G. H. M. Loewer-Sieger, D. H., 32 (V) Lommatzsch, P., 393 Luciano, L., 281

Maillard, G. F., 254 Medgyaszay, A., 73 Merenmies, L., 90 Möller, D. E., 411

Nádrai, A., 227 Niemi, A., 136 Nordmann, J., 129

Oosterhuis, J. A., 21 (V), 32 (V), 46 (V)

Paul, S. D., 150 Pawelski, W. J., 216 Pinckers, A., 232

440

Index autorum ad Vol. 163

Raab-Sternberg, A., 227 Radnót, Magda, 73 Raimondi, S., 325, 335 Rapola, J., 90 Raunio, V., 136 Reale, E., 281 Rempt, F., 209 Ronchi, Lucia, 189 Rutllan, J., 428 (B) 
Schappert-KimmijseГ, J., 12 (V) Schmidt, T., 120 Scouras, J., 254 Seddik, N., 325 Shimizu, K., 128 (B) Shinichi, S., 128 (B) Sie-Boen-Lian, 56 (V)

Singh, 150 Smith, B., 418 Sokolic, P., 356 Spitznas, M., 281

Tarkkanen, A., 90 Theodossiadis, G., 383 Thiel, R., 288 (B) Todt, H., 369

Van Balen, A. Th. M., 2 (V) , 8 (V) Van Lilh, G. H. M., 63 (V) Velický, J., 178

W. Worst

J.

$10(\mathrm{~V})$

Zauberman

$\mathrm{H}$.

65 